\title{
The value of inflow inversion recovery MRI for the diagnosis of transplant renal artery stenosis: comparison with digital subtraction angiography
}

\author{
Hongxia Li ${ }^{1}$, Guangrui Shao ${ }^{1}$, Yuan Zhao ${ }^{2}$ and Hai Zhong ${ }^{1 *}$ (1)
}

\begin{abstract}
Background: To date, few data on the assessment of transplant renal artery stenosis (TRAS) by using inflow inversion recovery (IFIR) are available. The aims of this study was to evaluate the feasibility of IFIR in the assessment of TRAS using Digital Subtraction Angiography (DSA) as the reference.

Results: We retrospectively assessed the IFIR of 195 transplant renal arteries. The IFIR images for 194/195 arteries were judged to be of excellent, good, or moderate quality, and 1/195 was not diagnostic. There were 100 arteries with TRAS, of which 27 were subjected to DSA. The stenosis percentages were divided into five grades. Using DSA images, the TRAS in 27 patients were estimated as grade $1(2,7.4 \%)$, grade $2(8,29.6 \%)$, grade $3(10,37.0 \%)$, grade $4(7,25.9 \%)$ and grade $5(0,0 \%)$. In comparison, the TRAS was shown to be grade $1(1,3.7 \%)$, grade $2(8,29.6 \%)$, grade $3(9,33.3 \%)$, grade $4(9,33 \%)$ and grade $5(0,0 \%)$ in the IFIR images. The nonparametric Wilcoxon signed-rank test was used to compare IFIR with DSA. In addition, a Bland-Altman plot was used to estimate the agreement between IFIR and DSA measurements. There was no significant difference between IFIR and DSA measurements $(p<0.05)$.

Conclusions: Relative to the reference DSA, IFIR was shown to be noninvasive, accurate for the diagnosis and evaluation of TRAS.

Keywords: Inflow inversion recovery, Transplant renal artery stenosis, Digital subtraction angiography, Computed tomographic angiography, Contrast-enhanced magnetic resonance angiography
\end{abstract}

\section{Background}

Renal transplantation is an effective treatment for endstage renal disease. In recent years, the total number of renal transplants has risen $[1,2]$. Although the improved surgical techniques have lowered the morbidity and mortality of renal transplant surgery, vascular postoperative complications still occur [3]. Among vascular complications, transplant renal artery stenosis (TRAS) is the most frequently observed and occurs in up to $23 \%$ of

\footnotetext{
*Correspondence: zhonghaifriend@126.com

1 Department of Radiology, the Second Hospital, Cheeloo College of Medicine, Shandong University, Jinan 250033, Shandong, China Full list of author information is available at the end of the article
}

cases, usually 3 months to 2 years after transplantation [4]. Most TRAS cases are prone to progress to transplant renal loss, which remains difficult to detect because of its nonspecific clinical manifestations. Therefore, early and accurate diagnosis of TRAS is essential [5].

Renal function recovery usually requires a long period of time after kidney transplantation. Moreover, to avoid rejection, recipients take several immunosuppressants, which increase the burden on renal function [6]. Digital Subtraction Angiography (DSA) is considered the gold standard for TRAS examination, but is invasive and expensive and requires the use of nephrotoxic iodinated contrast media; all factors limiting its clinical application. 
Two other imaging techniques, computed tomographic angiography (CTA) and contrast-enhanced magnetic resonance angiography (CE-MRA), have been used to assess the transplant renal vessels. Whereas CTA also requires the injection of iodinated contrast material [79], and exposure of the patient to radiation, nephrogenic systemic fibrosis may occur with the administration of linear gadolinium contrast agents for CE-MRA [10, 11]. Non-enhanced MRA includes phase contrast angiography, time of flight, electrocardiograph-gated fast spinecho, IFIR. Phase contrast angiography has long scanning time and low spatial resolution. No satisfactory imaging results have thus been reported. Time of flight has limitations with the long scan time, being sensitive to motion artifacts, and saturation of in-plane flow. In addition, because of the angle between the (transplanted) renal artery and the readout direction, it is difficult for electrocardiograph-gated fast spin-echo to show a clear vascular image [12]. Since Color-Doppler ultrasound examination is harmless and can be repeated in short intervals. Thus it can be used as a screening method [13], but caries a high false-positive rate and operator dependence. Therefore, there is an unmet need for an accurate and harmless diagnostic method for TRAS.

Inflow inversion recovery (IFIR) as a novel magnetic resonance imaging technology, has increasingly been applied to assess renal artery stenosis, with comparable results to those of DSA $[14,15]$. This technique does require no contrast agent and can therefore avoid kidney injury. Moreover, the short examination time of IFIR also allows for follow-ups. There has been so far little research using this technique to evaluate TRAS. In this study, IFIR was compared to DSA, which served as the gold standard for TRAS.

\section{Methods \\ Study population}

Between January 1, 2015 and December 31, 2018, we identified 165 kidney transplant patients (195 examinations, 158 men and 37 women, range 19-72 years old), who were suspected of having TRAS. The main reasons for patients for IFIR examination are as follows: (1) routine follow-up after kidney transplantation; (2) decreased renal function; (3) hypertension. All arteries were evaluated using IFIR as part of the routine clinical protocol. In the study, twenty-seven arteries with TRAS were evaluated with DSA. Among them, balloon dilatation was performed on nine patients, and balloon dilatation coupled with stent implantation was performed on the remaining 18 patients. The remaining patients did not undergo DSA for the following reasons: (1) no clinical symptoms or signs; (2) normal renal function/artery; (3) after clinical conservative treatment, satisfactory results were achieved. The study was approved by the ethics committees of our hospital (Table 1).

\section{MRI examination}

All IFIR measurements were performed using a $3.0 \mathrm{~T}$ whole-body scanner (Discovery 750, General Electrics (GE), Milwaukee, WI, USA). The standard MRI

Table 1 Demographic and summaries of study population $(n=195)$

\begin{tabular}{|c|c|c|c|c|}
\hline & $\begin{array}{l}\text { Whole cohort } \\
(n=195)\end{array}$ & $\begin{array}{l}\text { TRAS present } \\
(n=100,51.3 \%)\end{array}$ & $\begin{array}{l}\text { TRAS absent } \\
(n=95,48.7 \%)\end{array}$ & $\begin{array}{l}\text { DSA } \\
(n=27)\end{array}$ \\
\hline \multicolumn{5}{|l|}{ Age } \\
\hline Mean $\pm S D$ (year) & $42.0 \pm 10.8$ & $40.9 \pm 10.1$ & $43.2 \pm 11.5$ & $41.6 \pm 11.3$ \\
\hline Median (year) & 43 & 39 & 45 & 40 \\
\hline \multicolumn{5}{|l|}{ Sex } \\
\hline Male & $158(81.0 \%)$ & $83(83.0 \%)$ & $75(78.9 \%)$ & $22(81.5 \%)$ \\
\hline Female & $37(19.0 \%)$ & $17(17.0 \%)$ & $20(21.1 \%)$ & $5(18.5 \%)$ \\
\hline \multicolumn{5}{|c|}{ Location of renal transplant } \\
\hline Right iliac fossa & $183(93.8 \%)$ & $94(94.0 \%)$ & $89(93.7 \%)$ & $23(85.2 \%)$ \\
\hline Left iliac fossa & $12(6.2 \%)$ & $6(6.0 \%)$ & $6(6.3 \%)$ & $4(14.8 \%)$ \\
\hline \multicolumn{5}{|c|}{ Accessory renal artery } \\
\hline No & $185(94.9 \%)$ & $93(93.0 \%)$ & $92(96.8 \%)$ & $26(96.3 \%)$ \\
\hline Yes & $10(5.1 \%)$ & $7(7.0 \%)$ & $3(3.2 \%)$ & $1(3.7 \%)$ \\
\hline \multicolumn{5}{|c|}{ Overall image quality } \\
\hline Excellent & $162(83.1 \%)$ & $77(77.0 \%)$ & $85(89.5 \%)$ & $22(81.5 \%)$ \\
\hline Good & $24(12.3 \%)$ & $16(16.0 \%)$ & $8(8.4 \%)$ & $3(11.1 \%)$ \\
\hline Moderate & $8(4.1 \%)$ & $6(6.0 \%)$ & $2(2.1 \%)$ & $2(7.4 \%)$ \\
\hline Non diagnostic & $1(0.5 \%)$ & $1(1.0 \%)$ & $0(0.0 \%)$ & $0(0.0 \%)$ \\
\hline
\end{tabular}


protocol for kidney imaging started with an axial respirator-triggered single-shot fast spin-echo T2-weighted sequence and an axial breath-hold fast-spoiled gradient T1-weighted sequence. The imaging sequence was performed with breath-hold. The scan parameters were applied as follows: TE $1.5 \mathrm{~ms}$, TR $3.10 \mathrm{~ms}$, flip angle $60^{\circ}$, $\mathrm{TI}=1300 \mathrm{~ms}$, receiver bandwidth $125 \mathrm{~Hz} /$ pixel; field of view $340 \times 255 \mathrm{~mm}$ for covering both kidneys, slice thickness $2.4 \mathrm{~mm}$, locations per slab 32, frequency matrix 224, phase matrix 160, number of excitation 1, phase FOV 0.75 , and an acquisition time of $10-12 \mathrm{~s}$ [14].

\section{DSA examination}

DSA was performed with a GE DSA system (Innova; GE Healthcare, Waukesha, WI). Angiography was performed by experienced interventional radiologists through the femoral arterial route using a 5-F pigtail catheter to inject $30 \mathrm{ml}$ of iodinated contrast medium (Visipaque 320; Amersham Health, Princeton, NJ) at a flow rate of $15 \mathrm{ml} / \mathrm{s}$ (Mark V; Medrad, Indianola, PA). The optimal DSA images were obtained by $\mathrm{C}$ arm $\mathrm{X}$-ray roentgenscope in multiple directions and angles (frontal, left, and right oblique views). During the period between IFIR and DSA, the renal function of each patient was monitored and strictly controlled [16].

\section{Image analysis}

We used the scale measurement function/3D reconstruction/multiplanar reformation of the post-processing software (Discovery 750, General Electrics (GE), Milwaukee, WI, USA) to measure the diameter of arteries. The IFIR and DSA data were measured three times by two experienced radiologists separately. Mean value \pm standard deviation (SD) was taken of the IFIR and DSA data. Each radiologist completed the measurement independently. They were blinded to each other and they were blinded to relevant diagnosis before measurement.

The quality of IFIR images was assessed at four levels: excellent, good, moderate, and non-diagnostic (Fig. 1). The following measurements were made for each artery: The percentage of stenosis $=(1-(S / R)) \times 100$, where $S$ is the minimum diameter of the lesion and $R$ is the diameter of the reference site; the latter was defined as the normal-looking portion of the stenotic vessel distal or proximal to the lesion.

The extents of the TRAS were then graded as follows: grade $1(<25 \%)$; grade $2(25-49 \%)$; grade $3(50-74 \%)$; grade 4 (75-99\%); grade $5(100 \%)[14,17]$.

\section{Statistical analysis}

The data were analyzed in SPSS 17.0 statistical software and GraphPad Prism. The nonparametric Wilcoxon signed-rank test was used to compare IFIR to DSA of TRAS. The agreement between IFIR and DSA was assessed by Bland-Altman plots. The significance level was set to 0.05 .

\section{Results}

Our study included 165 patients, in which 138 patients underwent one IFIR examinations, 21 patients underwent two IFIR examinations at different time periods, and six patients underwent three IFIR examinations at different time periods. Each examination was independent. Three examinations were excluded from the group because of motion artifact. Therefore, 195 transplant renal arteries were eligible for inclusion during the study period. In the 195 arteries, there were 95 arteries without TRAS and 100 with TRAS. Twenty-seven arteries were also measured with DSA.

Patient demographics and summaries of the study are shown in Table 1. The age (mean $\pm \mathrm{SD} /$ median) of the whole cohort and those with TRAS was $42.0 \pm 10.8 / 43$ and $40.9 \pm 10.1 / 39$ years, respectively. Most of the renal transplants were in the right iliac fossa. Ten patients had accessory renal arteries, all of which showed in IFIR. The IFIR images were judged to be excellent (162, 83.1\%), good $(24,12.3 \%)$, moderate $(8,4.1 \%)$, and non diagnostic $(1,0.5 \%)$ (Table 1 and Fig. 1$)$.

Twenty-seven patients had undergone DSA, of which, all images of arteries were excellent (22, 81.5\%), good (3, $11.1 \%)$, and moderate quality $(2,7.4 \%)$ (Table 1$)$. In the DSA group, 2 TRAS were rated as grade 1,8 as grade 2, 10 as grade 3 , and 7 as grade 4 . In the IFIR group, 1,8 , 9, 9 TRAS was rated as grade $1-4$. There was no significant difference in stenosis percentage obtained by IFIR and DSA $(p=0.414)$ (Table 2). The Box plot (Fig. 2a) of percentage of stenosis detected by IFIR in patients with TRAS, by IFIR in patients with TRAS and by DSA was performed. The Bland-Altman plot (Fig. 2b) was performed, there was one sample $(3.7 \%,<5 \%)$ out of the $95 \%$ CI, which demonstrated strong consistency between DSA and IFIR. Compared to the DSA results, IFIR overestimated the transplant artery stenosis in four cases (Table 2, Fig. 3).

Since there was no contrast agent used in IFIR, followup measurements were performed. In Fig. 4, we showed images from three patients. Three IFIR examinations were performed at different times of each patient. The IFIR images were all judged to be excellent, and the renal artery and its distal branches were clearly displayed, which could meet the clinical assessment of arterial stenosis. In Fig. 5, we showed three patients who had IFIR measures before and after DSA examinations. The degree of arterial stenosis in image a (before DSA) and image b (DSA) showed a high consistency. After balloon dilation and/or stent implantation in image $c$, the degree of 

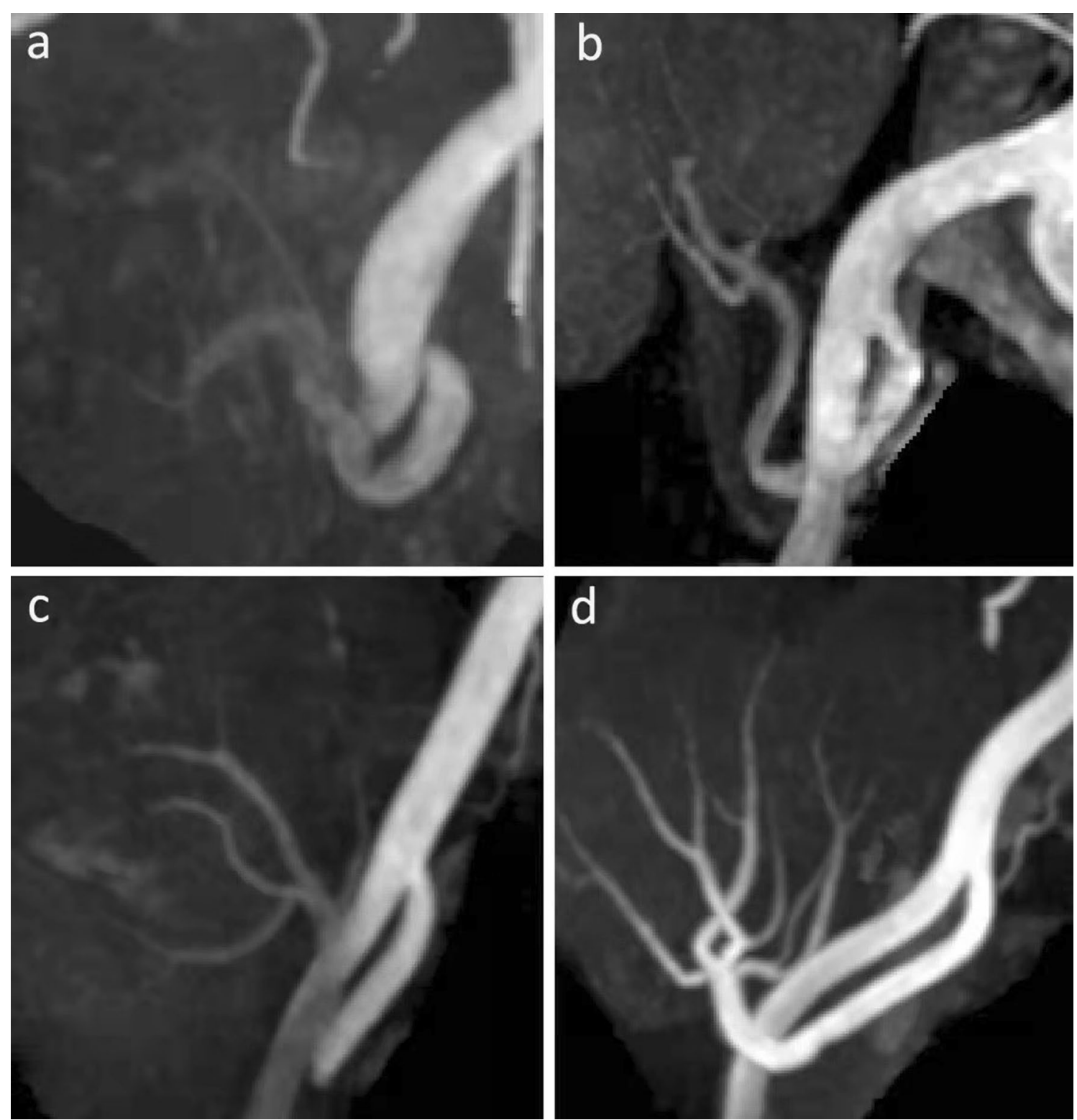

Fig. 1 Shows representative IFIR images of different quality: a non diagnostic, b moderate, c good, d excellent. IFIR: Inflow inversion recovery

Table 2 Twenty-seven patients had IFIR and DSA

\begin{tabular}{lll}
\hline Variables & DSA & IFIR-FIESTA \\
\hline Grade $1(<25 \%)$ & $2(7.4 \%)$ & $1(3.7 \%)$ \\
Grade 2 (25-49\%) & $8(29.6 \%)$ & $8(29.6 \%)$ \\
Grade 3 (50-74\%) & $10(37.0 \%)$ & $9(33.3 \%)$ \\
Grade 4 (75-99\%) & $7(25.9 \%)$ & $9(33.3 \%)$ \\
Grade 5 (100\%) & $0(0 \%)$ & $0(0 \%)$ \\
$n$ & 27 & 27 \\
$p$ & 0.414 & \\
\hline
\end{tabular}

Nonparametric Wilcoxon signed-rank test was performed, $p=0.414 . p<0.05$ indicated that the difference were statistically significant stenosis was significantly reduced. Image $d$ is a later follow-up picture, patient 1 and patient 2 were clearly displayed, and patient 3 was unclear due to stent artifacts.

\section{Discussion}

With the increasing number of kidney transplants performed, the prediction of transplant-related renal artery complications has become crucial. At present, both CTA and CE-MRA are widely applied techniques that provide excellent results in the assessment of transplant renal arteries [17]. However, it has been demonstrated that most drugs are excreted by the kidney, including the 
a

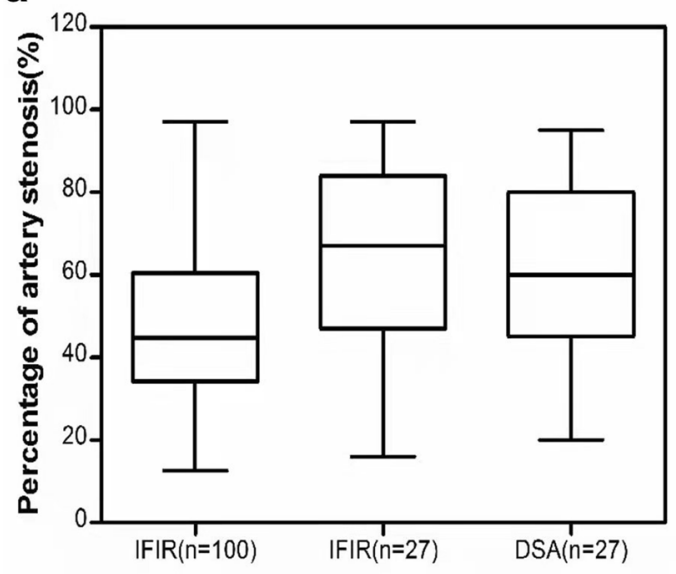

b

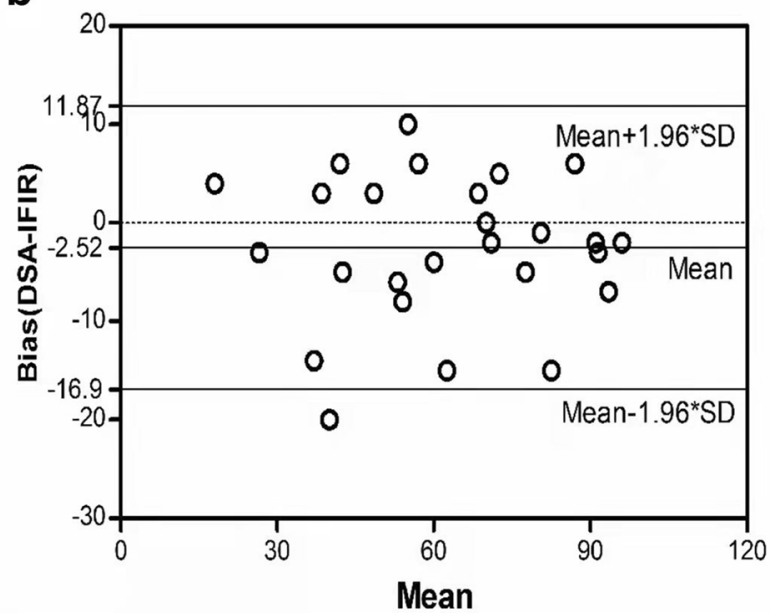

Fig. 2 a Box plot of the percentage of stenosis detected by IFIR in patients with TRAS ( $n=100)$, by IFIR $(n=27)$ in patients with TRAS and by DSA $(n=27)$. The $x$-axis represents groups, and the $y$-axis represents percentage of stenosis. $\mathbf{b}$ A Bland-Altman plot was performed, $95 \%$ confidence interval was -16.90 to 11.87 , and there was one sample $(3.7 \%,<5 \%)$ out of the $95 \% \mathrm{Cl}$, which demonstrated strong consistency between DSA and IFIR

iodine agent used for CTA examinations and gadolinium agent used for MRA examinations [18, 19]. Gadolinium may cause nephrogenic systemic fibrosis, which is a severe syndrome involving fibrosis of the internal organs, eyes, skin, and joints [18]. Therefore, iodine and gadolinium-based contrast methods used by CTA and MRA aggravate the burden on the kidney, which is undoubtedly not optimal for kidney transplant patients $[17,19]$.

In contrast, as a non-nephrotoxic imaging method, IFIR has attracted more attention in recent years. Because of these advantages (non-invasive examination, short scanning time, high signal intensity, and significantly suppressed background signals), IFIR can obtain clear images [14, 15, 17]. In our study, the IFIR images of 195 transplant renal arteries were assessed retrospectively. 194/195 IFIR images were diagnostic (excellent, 162; good, 24; moderate, 8 ), and 1/195 didn't have sufficient quality to be used in clinical diagnosis (non diagnostic, 1). There were 100 arteries with TRAS, and 27 arteries of them were also measured with DSA. For these arteries, the stenotic degrees measured by IFIR were highly consistent with the reference DSA, indicating high accuracy of IFIR in assessing TRAS. In research of Gaudiano C [14], the IFIR correctly assessed the grade in 17/19 stenoses, when compared with DSA. The finding was also discovered in our study. Moreover, IFIR could present the accessory renal artery as clearly as shown in DSA images, which demonstrated the ability of IFIR in displaying slim blood vessels. In research of Gaudiano C, 24 accessory renal arteries were displayed in the IFIR examination, and four accessory renal arteries were not displayed.
The detection rate is $85.7 \%$ [14]. In our study, a total of 10 accessory renal arteries were detected by IFIR. It can be concluded that in the IFIR examination, the accessory renal artery has a higher detection rate. This finding was also in agreement with other previous studies [20, 21]. In our study, 21 patients underwent two examinations and 6 patients underwent three examinations in different time. $59 / 60$ of the obtained images were of excellent/good quality, which could satisfy the clinical diagnosis. This also showed that IFIR is a good technique for follow-up after renal artery transplantation.

However, we also found that IFIR overvalued four cases of TRAS. The reasons could be explained as follows: a measurement error may lead to some measurement differences; the depiction of vessels could be affected by the filling state of vessels and the blood flow state. In particular, when the stenosis of the artery was severe, blood flow was fast and thus usually in a complex situation, resulting in nearby signal loss. The same result has also been confirmed in the study of Parienty et al. [21, 22]. Oppositely, in Nchimi A's study, IFIR underestimated two cases of renal arteries [13]. The reason for the underestimation may be measurement errors. However, this did not affect patients' clinical management.

Some limitations of this research should be mentioned. First, it is a retrospective study, the sample size receiving DSA measurements was small, which might introduce potential bias in patient selection. Secondly, due to the physical conditions of patients, the time interval between some IFIR and DSA examinations was more than seven days. There may be statistical 


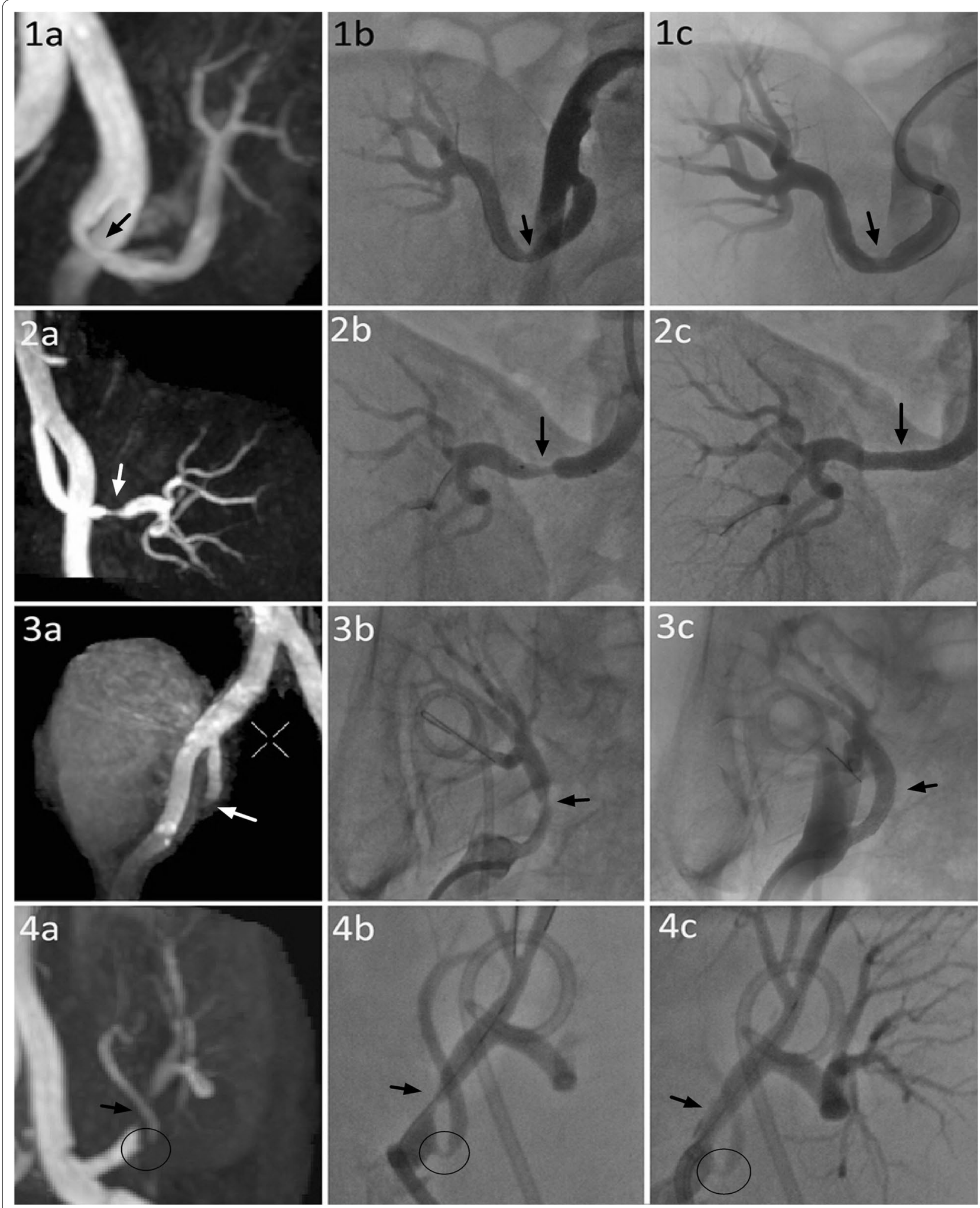

Fig. 3 Representative IFIR (a) and DSA images (b: pre-intervention, to confirm IFIR findings, c: after balloon dilatation and stent implantation) from four patients (1-4) with different degrees of TRAS. Patient 1-2: Stenosis degree of DSA images were the same with IFIR. Patient 3-4: IFIR images overestimated the stenosis. Patient $\mathbf{4 a - b}$ : An accessory renal artery was seen with initial stenosis, and IFIR produced a satisfactory concordance with DSA 


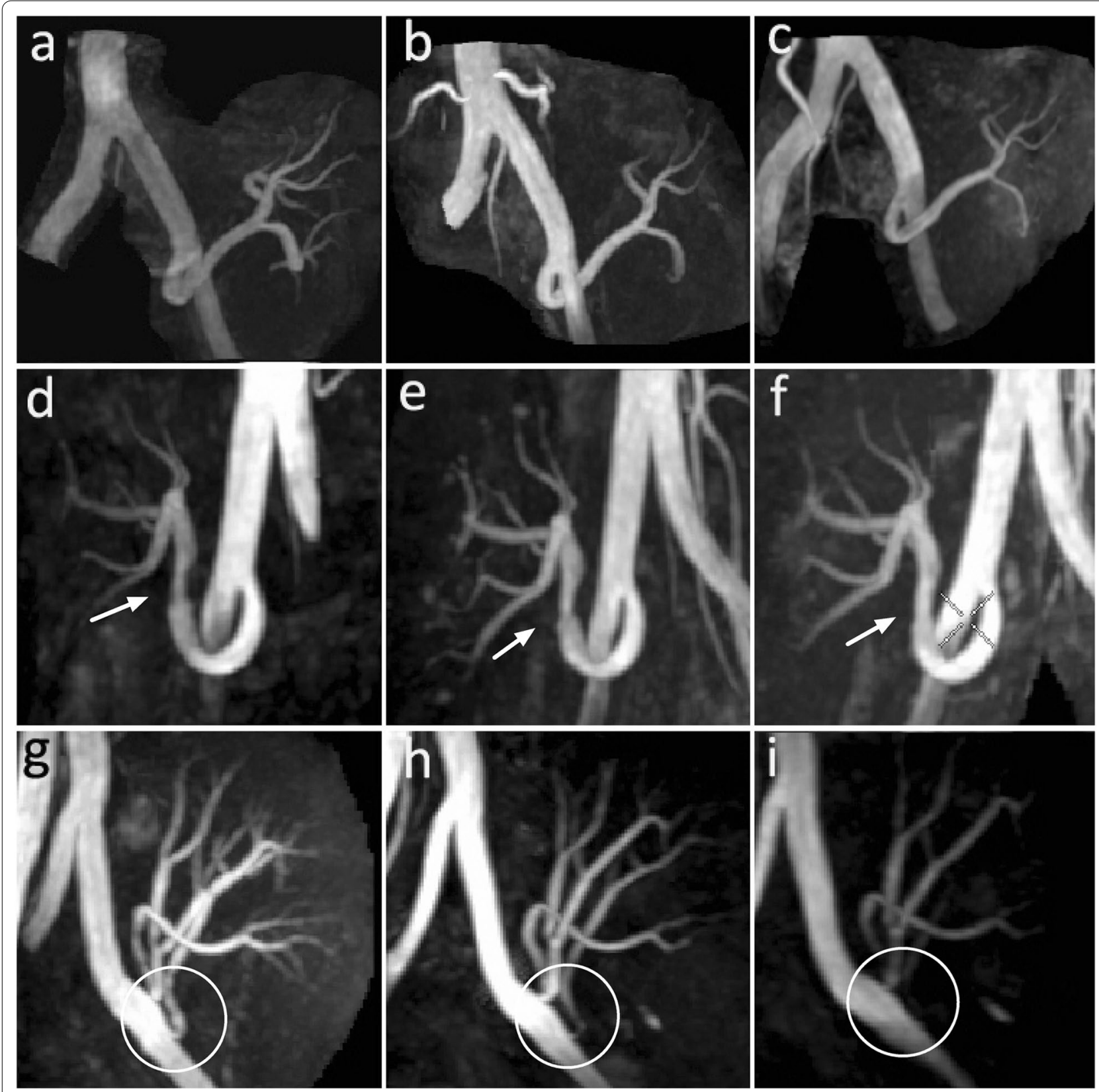

Fig. 4 Three IFIR examinations were performed in three patients. a, b, c A 49-year-old patient had 3 IFIR examinations on 2017-7-6, 2017-11-12, and 2018-6-4, respectively; d, e, f a 39-year-old patient had 3 IFIR examinations on 2016-4-26, 2016-5-17, and 2016-6-21, respectively; $\mathbf{g}$, h, i A 32-year-old patient had 3 IFIR examinations on 2016-6-30, 2016-8-2, and 2016-8-22, respectively, and an accessory renal artery was seen clearly in $\mathbf{g}$ h, i

errors in comparison of stenosis assessment between both modalities due to potential physical condition change. A more rigorous study is thus necessary to be performed to evaluate repeatability and reproducibility of IFIR assessments.

\section{Conclusions}

IFIR is noninvasive, accurate for the diagnosis and evaluation of TRAS and can be recommended as a valuable method in renal transplant patients for follow-up measurement. 

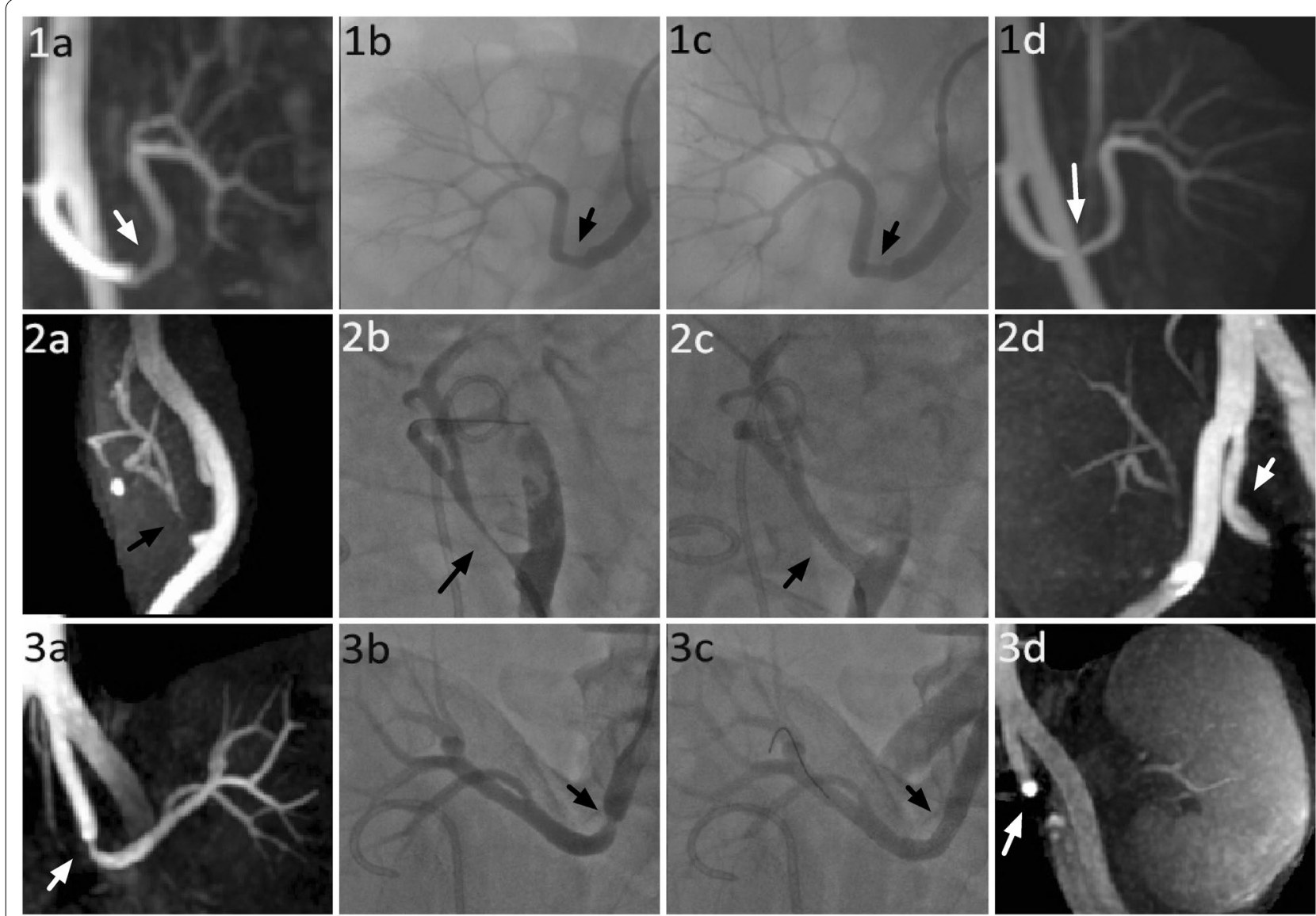

Fig. 5 Representative IFIR and DSA images from three patients (1-3) with different degrees of TRAS. IFIR images (a) before DSA showed arterial stenosis, and the extent of stenosis was demonstrated by DSA (b). Then, the balloon dilation and/or stent implantation was performed (c). IFIR examinations (Patient 1d) was performed after about 5 months, which showed no evident stenosis of the transplant renal artery. IFIR image (Patient 2d) showed that arterial stenosis still existed after balloon dilation and stent implantation about 1 year later. IFIR image (Patient 3d) cannot accurately display the degree of vascular stenosis due to the presence of artifacts about 3 months later

\section{Abbreviations}

IFIR: Inflow inversion recovery; FIESTA: Fast imaging employing steady-state acquisition; TRAS: Transplant renal artery stenosis; DSA: Digital subtraction angiography; CTA: Computed tomographic angiography; CE-MRA: Contrastenhanced magnetic resonance angiography; CDU: Color doppler ultrasound.

\section{Acknowledgements}

This work was supported by Department of Radiology, the Second Hospital, Cheeloo College of Medicine, Shandong University; Department of Interventional Medicine, the Second Hospital, Cheeloo College of Medicine, Shandong University; GE Healthcare, MR Research China. We thank International Science Editing (http://www.internationalscienceediting.com) for editing this manuscript.

\section{Authors' contributions}

$\mathrm{HL}, \mathrm{HZ}$ and GS were involved in the design and conception of the study. $\mathrm{HZ}$ and GS were responsible for the collection and analysis of the experimental data. HL drafted of manuscript. YZ contributed to reviewing/editing the manuscript. All authors read and approved the manuscript and agree to be accountable for all aspects of the research in ensuring that the accuracy or integrity of any part of the work are appropriately investigated and resolved.

\section{Funding}

This work was supported by China Health Promotion Foundation (XM_2020_011_0295_01).

\section{Availability of data and materials}

The datasets used and or analysed during the current study are available for the corresponding author on reasonable request.

\section{Declarations}

\section{Ethics approval and consent to participate}

All procedures performed in studies involving human participants were in accordance with the ethical standards of the institutional and/or national research committee and with the 1964 Helsinki declaration and its later amendments or comparable ethical standards. For retrospective studies formal consent is not required.

\section{Consent for publication}

For this type of study consent for publication is not required.

\section{Competing interests}

The authors declare that they have no competing interests. 


\section{Author details}

${ }^{1}$ Department of Radiology, the Second Hospital, Cheeloo College of Medicine, Shandong University, Jinan 250033, Shandong, China. ${ }^{2}$ Experimental Center, Shandong University of Traditional Chinese Medicine, Jinan 250355, Shandong, China.

Received: 13 October 2021 Accepted: 14 December 2021 Published online: 04 January 2022

\section{References}

1. Hart A, Smith JM, Skeans MA et al (2020) OPTN/SRTR 2018 annual data report: kidney. Am J Transplant 20(Suppl s1):20-130. https://doi.org/10. 1111/ajt.15672

2. Ammirati AL (2020) Chronic kidney disease. Rev Assoc Med Bras (1992) 66(Suppl 1):s03-s09. https://doi.org/10.1590/1806-9282.66.S1.3

3. Bashir S, Hussain M, Ali Khan A et al (2020) Renal transplant pathology: demographic features and histopathological analysis of the causes of graft dysfunction. Int J Nephrol 2020:7289701. https://doi.org/10.1155/ 2020/7289701

4. Bruno S, Remuzzi G, Ruggenenti P (2004) Transplant renal artery stenosis. J Am Soc Nephrol 15:134-141. https://doi.org/10.1097/01.asn.00000 99379.61001.f8

5. Adani GL, Como G, Bonato F et al (2018) Detection of transplant renal artery stenosis with contrast-enhanced ultrasound. Radiol Case Rep 13:890-894. https://doi.org/10.1016/j.radcr.2018.06.003

6. Tsai JL, Tsai SF (2020) Recovery of renal function in a kidney transplant patient after receiving hemodialysis for 4 months. Exp Clin Transplant 18:112-115. https://doi.org/10.6002/ect.2017.0323

7. Wang L, Zhu L, Li G, Zhang Y et al (2021) Gadolinium-enhanced magnetic resonance versus computed tomography angiography for renal artery stenosis: a systematic review and meta-analysis. J Formos Med Assoc 11:S0929-6646(21)00024-3. https://doi.org/10.1016/j.jfma.2021.01.007

8. Helck A, Bamberg F, Sommer WH et al (2011) Optimized contrast volume for dynamic CT angiography in renal transplant patients using a multiphase CT protocol. Eur J Radiol 80:692-698. https://doi.org/10.1016/j. ejrad.2010.10.010

9. Sadowski EA, Bennett LK, Chan MR et al (2007) Nephrogenic systemic fibrosis: risk factors and incidence estimation. Radiology. https://doi.org/ 10.1148/radiol.2431062144

10. Kitajima K, Maeda T, Watanabe S, Ueno Y, Sugimura K (2012) Recent topics related to nephrogenic systemic fibrosis associated with gadoliniumbased contrast agents. Int J Urol 19:806-811. https://doi.org/10.1111/j. 1442-2042.2012.03042.x

11. Weinreb JC, Kuo PH (2009) Nephrogenic systemic fibrosis. MagnReson Imaging Clin N Am 17:159-167. https://doi.org/10.1016/j.mric.2009.01. 003

12. Morita S, Masukawa A, Suzuki K, Hirata M, Kojima S, Ueno E (2011) Unenhanced MR angiography: techniques and clinical applications in patients withchronic kidney disease. Radiographics 31:E13-E33. https://doi.org/ 10.1148/rg.312105075

13. Nchimi A, Biquet JF, Brisbois D et al (2003) Duplex ultrasound as first-line screening test for patients suspected of renal artery stenosis: prospective evaluation in high-risk group. Eur Radiol 13:1413-1419. https://doi.org/ 10.1007/s00330-002-1685-7

14. Gaudiano C, Busato F, Ferramosca E et al (2014) 3D FIESTA pulse sequence for assessing renal artery stenosis:is it a reliable application in unenhanced magnetic resonanceangiography? Eur Radiol 24:3042-3050. https://doi.org/10.1007/s00330-014-3330-7

15. Yamuna J, Chandrasekharan A, Rangasami R, Ramalakshmi S, Joseph S (2017) Unenhanced renal magnetic resonance angiography in patients with chronic kidney disease \& suspected renovascular hypertension: Can it affect patient management? Indian J Med Res 146:S22-S29. https://doi. org/10.4103/ijmr.IJMR_1613_15

16. Parienty I, Rostoker G, Jouniaux F, Piotin M, Admiraal-Behloul F, Miyazaki M (2011) Renal artery stenosis evaluationin chronic kidney diseasepatients: nonenhanced time-spatial labeling inversion-pulsethree-dimensional MR angiography with regulated breathing versus DSA. Radiology 259:592601. https://doi.org/10.1148/radiol.11101422
17. Tang H, Wang Z, Wang L et al (2014) Depiction of transplant renal vascular anatomy and complications: unenhanced MR angiography by using spatial labeling with multiple inversion pulses. Radiology 271:879-887. https://doi.org/10.1148/radiol.14131800

18. Rogosnitzky M, Branch S (2016) Gadolinium-based contrast agent toxicity: a reviewof known and proposed mechanisms. Biometals 29:365-376. https://doi.org/10.1007/s10534-016-9931-7

19. Lusic H, Grinstaff MW (2013) X-ray computed tomography contrast agents. Chem Rev 113:1641-1666. https://doi.org/10.1021/cr200358s

20. Zhang L, Peng J, Wen J et al (2018) Non-contrast-enhanced magnetic resonance angiography: a reliable clinical tool for evaluating transplant renal artery stenosis. Eur Radiol 28(10):4195-4204. https://doi.org/10. 1007/s00330-018-5413-3

21. Parienty I, Rostoker G, Jouniaux F, Piotin M, Admiraal-Behloul F, Miyazaki M (2011) Renal artery stenosis evaluation in chronic kidney disease patients: nonenhanced time-spatial labeling inversion-pulse three-dimensional MR angiography with regulated breathing versus DSA. Radiology 259(2):592-601. https://doi.org/10.1148/radiol.11101422

22. Guo X, Gong Y, Wu Z, Yan F, Ding X, Xu X (2020) Renal artery assessment with non-enhanced MR angiography versus digital subtraction angiography: comparison between 1.5 and 3.0 T. Eur Radiol 30(3):1747-1754. https://doi.org/10.1007/s00330-019-06440-0

\section{Publisher's Note}

Springer Nature remains neutral with regard to jurisdictional claims in published maps and institutional affiliations.

\section{Submit your manuscript to a SpringerOpen ${ }^{\circ}$ journal and benefit from:}

- Convenient online submission

- Rigorous peer review

- Open access: articles freely available online

- High visibility within the field

- Retaining the copyright to your article

Submit your next manuscript at $\boldsymbol{\nabla}$ springeropen.com 